\title{
FLEXIBLE ALGEBRAS OF DEGREE ONE
}

\author{
ERWIN KLEINFELD AND LOUIS A. KOKORIS ${ }^{1}$
}

1. Introduction. Let $A$ be a simple, flexible, powerassociative, finite-dimensional algebra over a field of characteristic zero. Then it is known that $A$ has a unity element 1 [5], and consequently $A$ has a degree. When $A$ has degree larger than two, Oehmke has shown [5] that $A^{+}$is a simple Jordan algebra. Kokoris [4] has shown the same result in case $A$ has degree two. In this paper we are able to show that if $A$ has degree one then in fact $A$ must be a one-dimensional algebra. Combining these results, the following theorem may be asserted.

Main Theorem. If $A$ is a simple, flexible, powerassociative, finitedimensional algebra of characteristic zero then $A^{+}$is a simple Jordan algebra.

2. Proof. We begin with a result that is more general than actually needed to prove the main theorem.

Theorem 1. Let $R$ be a flexible algebra with unity element 1 over a field $F$ of characteristic not two. Suppose there exists some vector space decomposition of $R, R=F 1+N$, such that for all elements $a, b$ in $N$ $a \cdot b=(a b+b a) / 2$ is in $N$. Then the ideal $C$ generated by all elements of the form $(x, y, z)=(x \cdot y) \cdot z-x \cdot(y \cdot z)$ is contained in $N$ and hence is $a$ proper ideal of $R$.

PROOF. For arbitrary elements $x_{1}, x_{2}, y$ in $N$ we have $x_{1} y=\lambda_{1} 1+z_{1}$, and $x_{2} y=\lambda_{2} 1+z_{2}$, where $z_{1}$ and $z_{2}$ are in $N$, while $\lambda_{1}, \lambda_{2}$ are scalars. As in Schafer [7, Relation (8)] it follows from the flexible law that

$$
\begin{aligned}
\left(x_{1} \cdot x_{2}\right) y= & \lambda_{1} x_{2}+\lambda_{2} x_{1}+x_{1} \cdot z_{2}+x_{2} \cdot z_{1}-\left(x_{1} \cdot y\right) \cdot x_{2}-\left(x_{2} \cdot y\right) \cdot x_{1} \\
& +\left(x_{1} \cdot x_{2}\right) \cdot y .
\end{aligned}
$$

As in Kokoris [3, p. 653] one goes on to show from (1) that

$$
\begin{aligned}
\left(x_{1}, x_{2}, x_{3}\right) y= & \left(x_{1}, x_{2}, z_{3}\right)+\left(x_{1}, z_{2}, x_{3}\right)+\left(z_{1}, x_{2}, x_{3}\right)-\left(x_{1} \cdot y, x_{2}, x_{3}\right) \\
& -\left(x_{1}, x_{2} \cdot y, x_{3}\right)+\left(x_{3} \cdot y, x_{2}, x_{1}\right)+\left(x_{1}, x_{2}, x_{3}\right) \cdot y
\end{aligned}
$$

where $(x, y, z)$ is defined here as $(x, y, z)=(x \cdot y) \cdot z-x \cdot(y \cdot x)$, while $x_{3} y=\lambda_{3} 1+z_{3}$, where $z_{3}$ is in $N$ and $\lambda_{3}$ is a scalar. Then if $B$ is the subspace generated by all $(x, y, z)$, relation (2) implies that $B N \subset B$

Received by the editors August 16, 1961.

${ }^{1}$ One of the authors was supported in part by a grant from the Office of Ordnance Research. 
$+B \cdot N, \quad N B \subset B+B \cdot N$, and more generally $((B N) \cdots) N \subset B$ $+B \cdot N+\cdots+((B \cdot N) \cdots) \cdot N$ etc. As a result the set $C$, defined as the set of all finite sums of elements from the sets $B, B \cdot N$, $(B \cdot N) \cdot N, \cdots$, can be shown to be an ideal of $A$. Since $B$ is readily shown to be in $N$ and since $N \cdot N \subset N$ by hypothesis, we may conclude that $C \subset N$. This concludes the proof of the theorem.

Corollary. If $R$ is also assumed to be simple then $R^{+}$is an associative, commutative algebra.

While the following theorem is not essential to the proof of the Main Theorem, it together with Theorem 1 might be useful in a study of flexible algebras where the elements of $N$ are not necessarily nilpotent.

THEOREM 2. If $S$ is a flexible ring of characteristic different from two such that $S^{+}$is powerassociative, then $S$ must be powerassociative.

Proof. From the flexible law third-power associativity follows. Assume inductively $k$-power associativity for all $k<n$. We proceed to establish $n$-power associativity. The flexible law implies that

$$
x^{n-1} x=\left(x x^{n-2}\right) x=x\left(x^{n-2} x\right)=x x^{n-1} .
$$

By a second induction suppose $x^{n-a} x^{a}=x^{a} x^{n-a}$ for $0<a<n-1$. We have already established this for $a=1$. The linearized form of the flexible law implies that

$$
\left(x^{n-a-1} x\right) x^{a}+\left(x^{a} x\right) x^{n-a-1}=x^{n-a-1}\left(x x^{a}\right)+x^{a}\left(x x^{n-a-1}\right) .
$$

By the second induction hypothesis the first term on the left cancels the second term on the right in the last equality, leaving

$$
x^{n-(a+1)} x^{(a+1)}=x^{(a+1)} x^{n-(a+1)} .
$$

This completes the proof of the second induction. Powerassociativity in $A^{+}$implies

$$
\left(x^{a} \cdot x^{n-a-1}\right) \cdot x=x^{a} \cdot\left(x^{n-a-1} \cdot x\right) .
$$

However from this it follows that

$$
2 x^{n-1} \cdot x=2 x^{n-a} \cdot x^{a},
$$

so that, for all $a, x^{n-1} \cdot x=x^{n-a} \cdot x^{a}$, assuming characteristic different from two. This completes the first induction and the proof of the theorem.

We note that in general powerassociativity of $T^{+}$does not suffice to guarantee powerassociativity of $T$. 
CoROllary. If $R$ is simple then $R$ must be powerassociative.

Consider now the case at hand, in which $A$ is assumed to have degree one over an algebraically closed field. Then there exists a vector space decomposition $A=F 1+N$, where in fact all elements of $N$ are nilpotent. Albert [2, p. 527] has shown that in $A^{+}, N$ is a subalgebra. From this one infers that $A$ satisfies the hypotheses of Theorem 1. From the Corollary to Theorem 1 it follows that $A^{+}$is associative. Hence $A$ is a noncommutative Jordan algebra. At this point a result of Schafer's [6, Main Theorem] may be used to conclude that $A$ is trace-admissible. Albert [1, Principal Theorem] has shown that a trace-admissible algebra $A$ is simple if and only if $A^{+}$is simple. Thus $A^{+}$is a simple, associative, commutative, finite-dimensional algebra. Then it is well known that $A^{+}$must be a field. Therefore $N$ must be zero. This of course means $A$ is isomorphic to $F$. We have proved

Theorem 3. If $A$ is a simple, flexible, powerassociative, finite-dimensional algebra over an algebraically closed field of characteristic zero and degree one then $A$ is a one dimensional field.

The existence of nodal, noncommutative Jordan algebras indicates that the conclusion of Theorem 3 is not true for fields of finite characteristics [3].

\section{REFERENCES}

1. A. A. Albert, $A$ theory of trace-admissible algebras, Proc. Nat. Acad. Sci. U.S.A. 35 (1949), 317-322.

2. - A theory of powerassociative, commutative algebras, Trans. Amer. Math. Soc. 69 (1950), 503-527.

3. L. A. Kokoris, Simple nodal noncommutative Jordan algebras, Proc. Amer. Math. Soc. 9 (1958), 652-654.

4. - Flexible nilstable algebras, Proc. Amer. Math. Soc. 13 (1962), 335-340.

5. R. H. Oehmke, On flexible algebras, Ann. of Math. 68 (1958), 221-230.

6. R. D. Schafer, Noncommutative Jordan algebras of characteristic zero, Proc. Amer. Math. Soc. 6 (1955), 472-475.

7. - On noncommutative Jordan algebras, Proc. Amer. Math. Soc. 9 (1958), 110-117.

Ohio State University AND

ILlinois INSTITUTE of TEChNOLOGY 